\title{
Platelet-rich plasma combined with core decompression and al- logeneic fibula rod support for the treatment of osteonecrosis of the femoral head (Ficat stage II): a short-term follow-up
}

\author{
Liang-bin Jiang, ${ }^{\mathrm{a}, \mathrm{b}}$, Song Liu ${ }^{\mathrm{a}, \mathrm{b}}$, Biao-fang Wei ${ }^{\mathrm{b}}$ \\ ${ }^{a}$ Guang zhou University of Chinese Medicine $;{ }^{b}$ Department of Orthopaedic Surgery, Lin Yi People's Hospital
}

\begin{abstract}
Background: The objective of this study was to investigate the potency of platelet-rich plasma (PRP) combined with core decompression and allogeneic fibula rod support for the treatment of osteonecrosis of the femoral head (ONFH, Ficat stage II).

Methods: This was a retrospective study of clinical data of patients who were admitted to our hospital for treatment of ONFH from July 2014 to June 2015. In PRP group, patients ( 9 cases) were treated with plateletrich plasma combined with core decompression and allogeneic fibula rod support. In control group, patients (7 cases) were treated with core decompression and allogeneic fibula rod support only. The time from onset of symptoms to surgery was 3-6 months (mean: 4.5 months). Harris hip scores before and after surgery were recorded (> 90, excellent; 75-90, good; 60-74, poor; and <60, bad). An imaging study was performed 1 and 3 months after surgery.

Results: In the PRP group, the Harris scores increased from $67.82 \pm 9.61$ to $88.45 \pm 6.02$. In the control group, the Harris scores increased from $69.74 \pm 8.26$ to $87.36 \pm 6.17$. There were statistically significant differences in pretherapy and post-treatment scores $(\mathrm{P}<0.05)$. Three months postsurgery, the shapes of the femoral heads in both groups were good, with no deformation or collapse. The location of the top of the allograft fibula was perfect (no fibula out). Additionally, the fibula rods began to combine with the surrounding bone after 3 months postsurgery, without infection.
\end{abstract}

Conclusion: PRP can ease hip pain and improve joint function

Keywords: PRP, ONFH, core decompression, allogeneic fibula rod

\section{INTRODUCTION}

Osteonecrosis of the femoral head (ONFH) is a painful disorder of the hip that affects patients with various risk factors, such as glucocorticoid use, alcohol abuse, trauma, and autoimmune diseases ${ }^{(1-3)}$. The progress of joint destruction in ONFH can lead to hip arthritis, which seriously affects the patient's health. In the absence of appropriate surgical treatment, the femoral head will collapse within 2-3 years in the vast majority of patients ${ }^{(4)}$. Steinberg ${ }^{(5)}$ reported that collapse of the femoral head occurred in $92 \%$ of nonsurgical cases $(48$ hips) within 3 years after a definite diagnosis of ONFH.

\footnotetext{
*Corresponding author: Biao-fang Wei

Address: Department of Orthopaedic Surgery, Lin Yi People's

Hospital, No.27 East Jiefang Road, Linyi, 276000

E-mail: 317516288@qq.com

Received: 20 October 2017 Accepted: 25 December 2017
}

As ONFH occurs mostly in young and middle-aged patients, Garrigues et al. (6) believe that all possible treatment measures should be taken to protect the femoral head. At present, the main methods to protect the femoral head in ONFH are core decompression, with or without a bone graft, in addition to bone flap transplantation with blood vessels and blood vessel bundle transplantation. However, these treatments have not achieved satisfactory clinical results.

Platelet-rich plasma (PRP), an autologous enriched source of various growth factors, is widely used during trauma and orthopedic surgery. PRP contains a variety of cytokines, such as platelet-derived growth factor (PDGF), vascular endothelial growth factor, plateletderived angiogenesis factor, transforming growth factor (TGF), insulin-like growth factor, and plateletderived epidermal growth factor. PRP can accelerate the healing of hard and soft tissues after maxillofacial, plastic, dermatologic, dental, and orthopedic surgery 
(7-9). However, evidence from preclinical and clinical research suggests that PRP has no significant therapeutic effects on ONFH. The aim of the present study was to investigate whether PRP promoted healing of ONFH in a clinical setting.

\section{MATERIAL AND METHODS}

\section{Patients}

This was a retrospective study of clinical data of patients who were admitted to our hospital for treatment of ONFH from July 2014 to June 2015. The patients were assigned to a PRP group or a control group. In the PRP group, there were seven males and two females aged 28-45 years (mean: 36.5 years). The group included six cases of alcoholic avascular necrosis and three cases of glucocorticoid-induced avascular necrosis. The Harris score was 60-86 points (mean: 73 points) prior to surgery. Patients were treated with plateletrich plasma combined with core decompression and allogeneic fibula rod support. In the control group, there were five males and two females aged 26-44 years (mean: 35 years). The group contained four cases of alcoholic avascular necrosis, two cases of glucocorticoid-induced avascular necrosis, and one case of traumatic ONFH. The Harris score was 62-86 points (mean: 74 points) prior to surgery. Patients were treated with core decompression and allogeneic fibula rod support only. In all patients, ONFH was confirmed by symptoms, a physical examination, patient history, and results of imaging studies, including X-ray and magnetic resonance (MR) examinations. The time from onset of symptoms to surgery was 3-6 months (mean: 4.5 months). There were no statistically significant differences in the common data between the two groups $(\mathrm{P}>0.05)$. The clinical experiments were approved by the ethics committee of Linyi People's Hospital. All the patients volunteered to take part in the study, and all signed informed consent forms.

\section{Preparation of PRP}

After successful induction of general anesthesia, whole blood (30 mL) was collected from the marginal auricular vein using an 18-gauge catheter. The blood was then injected into a sterile centrifuge tube containing 1.5 $\mathrm{mL}$ of sodium citrate. The mixture was centrifuged at $1500 \times \mathrm{g}$ for $20 \mathrm{~min}$ to separate the plasma from red blood cells. A straw was then used to suck out the supernatant. The plasma was centrifuged again at $1500 \times \mathrm{g}$ for $10 \mathrm{~min}\left(4^{\circ} \mathrm{C}\right)$, and the precipitated platelets (i.e., PRP) were collected (10).

\section{Surgical procedure}

A longitudinal incision (4-6 cm) was made in the big tuberosity distal along the lateral femoral hip to expose the far side of the greater trochanter of the femur, which was used as the entry point. Using C-arm X-ray machine monitoring, a bone tunnel (diameter of 12 $\mathrm{mm}$ ) was drilled in the central necrosis area (5-7 mm under the articular cartilage). All dead bone tissue was then removed along the bone tunnel, and autologous PRP was implanted. Selecting suitable length allogeneic fibula rod support and fixed. After confirming the depth and location of allogeneic fibula rod, the incision was sutured. The surgical protocol is shown in Figure 1.

\section{Postoperative management}

All the patients were treated with anti-inflammatory agents after the surgery. After awakening from anesthesia, active flex and outreach of joint function rehabilitation exercise was carried out. Postsurgery, the patients were supported by crutches for 3 months to avoid femur neck fracture caused by falling. Each
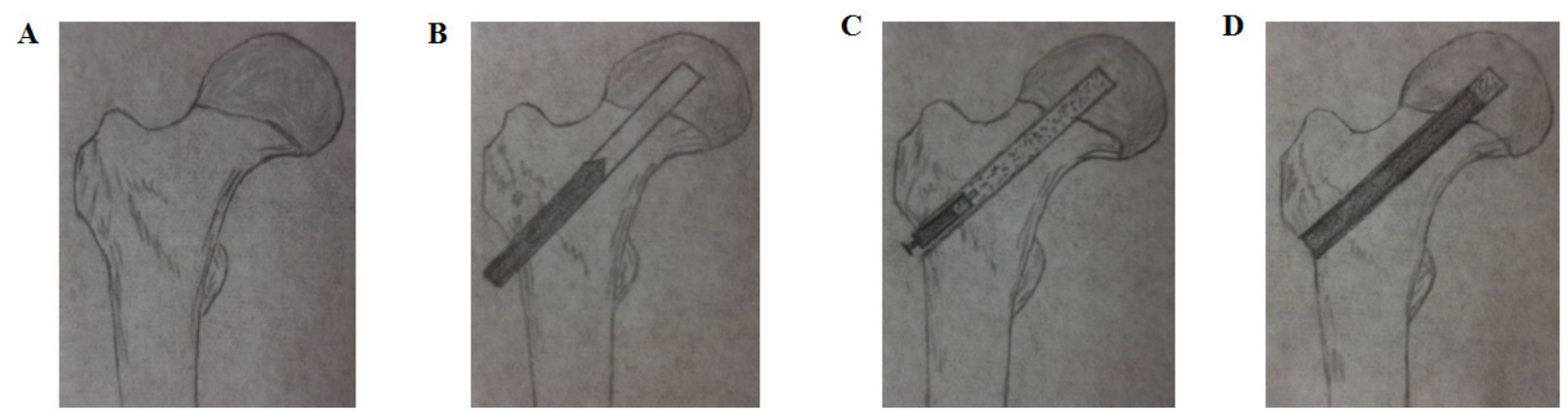

Figure 1. Surgical procedure. (A) Step 1: Identifying the location of ONFH. (B) Step 2: Construction of a bone tunnel and removal of dead bone tissue. (C) Step 3: Implantation of autologous PRP. (D) Step 4: Selection of an allogeneic fibula rod support of a suitable length and fixation. The anesthesia method, incision approach, and operative procedure in the control group were the same as those in the PRP group, except PRP was not implanted in the control group. 
Table 1 Comparison of Harris hip scores in the two groups before and after treatment

\begin{tabular}{lllll}
\hline Group & $\mathbf{n}$ & Before treatment & Final follow-up & P \\
\hline PRP group & 9 & $67.82 \pm 9.61$ & $88.45 \pm 6.02$ & $<0.05$ \\
Control group & 7 & $69.74 \pm 8.26$ & $87.36 \pm 6.17$ & $<0.05$ \\
P & $>0.05$ & $>0.05$ & \\
\hline
\end{tabular}

patient was advised to avoid excessive body weight and intense activity for 1 year.

Postoperative follow-up and evaluation of the curative effect

As an indicator of the curative effect, the Harris hip score was used (> 90, excellent; 75-90, good; 60-74, poor; and $<60$, bad) ${ }^{(11)}$. Imaging studies were performed 1 and 3 months after the surgery to evaluate changes in bone morphology.

\section{Statistical analysis}

All data are expressed as means \pm standard deviation (SD). The statistical analysis was performed using SPSS 16.0 software (SPSS Inc., Chicago, IL, USA). Differences between groups were detected using a one-way analysis of variance, followed by Scheffe's multiple comparison test. $\mathrm{P}<0.05$ was considered statistically significant.

\section{RESULTS}

Comparison of Harris hip scores in the two groups

In the PRP group, the Harris scores increased from $67.82 \pm 9.61$ to $88.45 \pm 6.02(\mathrm{P}<0.05$, Table 1$)$. Seven (77.8\%) hips were classed as excellent, and one (22.2\%) hip was classed as good. No hips were classed as poor or bad. In the control group, the Harris scores increased from $69.74 \pm 8.26$ to $87.36 \pm 6.17(\mathrm{P}<0.05$, Table 1$)$. Six (85.7\%) hips were classed as excellent, and 1 (14.2\%) hip was classed as good. No hips were classed as poor or bad. There were statistically significant differences in pretreatment and post-treatment scores $(\mathrm{P}<$ 0.05 , Table 1). There were no statistically significant differences in the postoperative scores of the two groups ( $\mathrm{P}>0.05$, Table 1$)$.

\section{Imaging findings}

Three months after the operation, the shapes of the femoral heads in the two groups were good, with no deformation and collapse. The location of the top of the allograft fibula was perfect (no fibula out), and it was located in the weight-bearing area and 5-7 $\mathrm{mm}$ (mean $6 \mathrm{~mm}$ ) under the articular cartilage. The fibula rods began to combine with the surrounding bone after 3 months postsurgery, without infection. There were no statistically significant differences in the imaging findings of the two groups (Fig. 2).

\section{DISCUSSION}

The purpose of this study was to evaluate the therapeutic effect of PRP in ONFH (Ficat stage II). In the initial stage of $\mathrm{ONFH}$, core decompression, together with allogeneic fibula rod support, is considered an effective surgical intervention. However, However, this treatment retains the femoral head in only $60-80 \%$ of cases in the short and medium term ${ }^{(12)}$. Femoral head core decompression involves drilling a tunnel to reduce the pressure on the femoral head, thereby alleviating pain. A combination of core decompression with other treatments, such as a bone graft and implants, with growth factors, can promote local osteogenesis and avoid channel collapse. The ultimate aim is to reduce pressure in the necrotic area, remove necrotic tissue, and provide effective mechanical support. The clinical application of PRP in the treatment of early-stage ONFH offers a new strategy to combat disease progression. The platelet (PLT) concentration in PRP is several times higher than that in whole blood, although findings differ on this issue. Marx et al. ${ }^{(13)}$ reported that the concentration of PLT in PRP was four times higher than that in whole blood. In contrast, Okuda et al. ${ }^{(14)}$ concluded that the concentration of PLT in PRP was 2.83 times higher than that in whole blood. Others reported that the concentration of PLT in PRP was 2.05 (15), 5.05 (artificial count) ${ }^{(15)}, 5.29$ (automated account) (16), and even 9-11 times higher as compared with that in whole blood ${ }^{(17)}$.

PRP-induced bone regeneration is mainly due to growth factors, which are released in response to activation of PRP (18-20). Growth factors, especially PDGF and TGF- $\beta$ play a vital role in the process of bone regeneration and repair ${ }^{21)}$. In addition to growth factors in PLTs, macrophages and endothelial cells, which synthesize and secrete PDGF, promote wound healing (22). PDGF promotes mitosis and related angiogenesis (23) and 

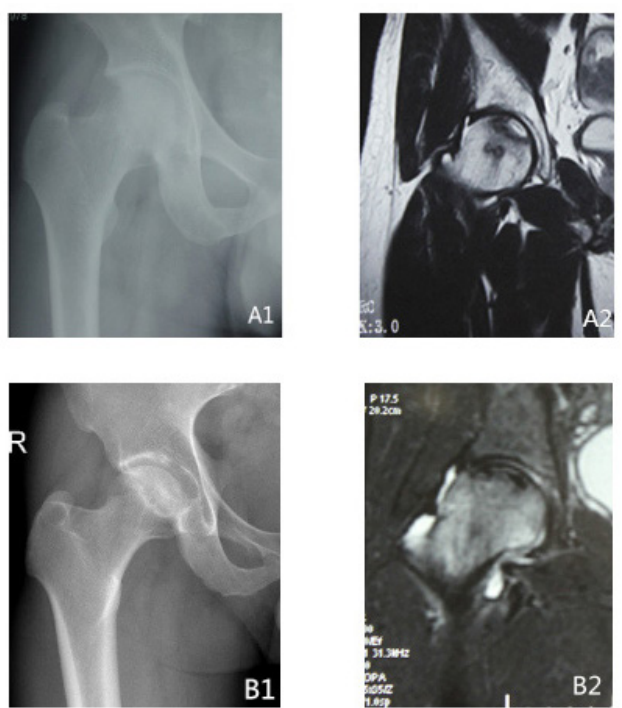

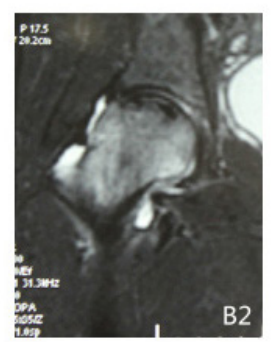

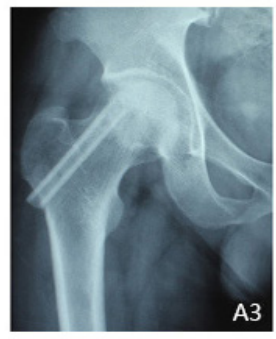
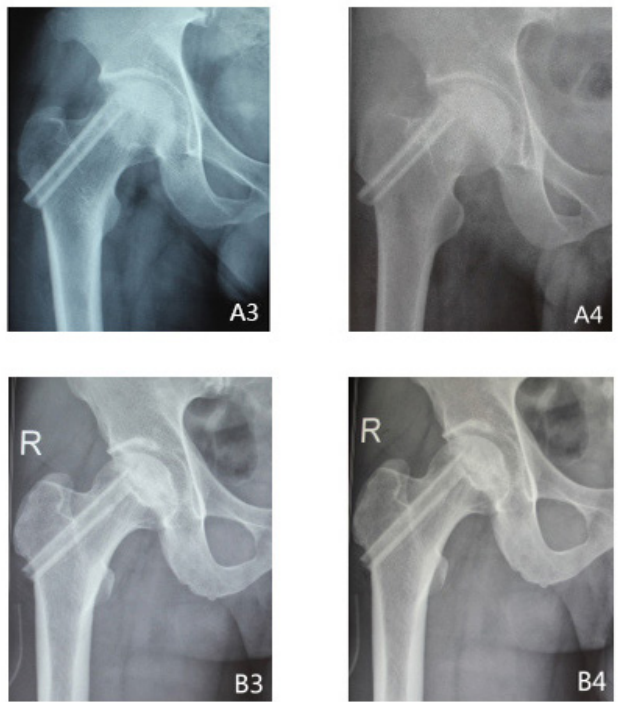

Figure 2. Imaging findings. (A1 and B1) AP X-ray films before surgery. (B1 and B2) Coronal T1 magnetic resonance (MR) images before surgery. (A3\&B3) AP X-ray films after surgery. (A4 and B4) AP X-ray films 3 months after surgery.

improves the activity of macrophages and other growth factors. TGF- $\beta$ induce mitosis, osteoblast differentiation, osteoid formation, and bone regeneration (24) . nsulin-like growth factor (IGF) accelerates bone formation by increasing the number of osteoblasts ${ }^{25)}$. To shed light on the therapeutic effect of growth factors, Lynch et al. ${ }^{26)}$ used different concentrations of PDGF, IGF, epidermal growth factor (EGF), and Fibroblast growth factor (FGF) mixtures to repair wounds and found that a 2:1 ratio of PDGF and IGF was the best combination. They suggested that a variety of growth factors constitute a growth factor net and have a synergistic effect. For example, combined PDGF and TGF- $\beta$ had a strong biological chemotactic effect on human osteoblasts ${ }^{(27)}$. TGF- $\beta$ not only regulated growth and differentiation of bone cells and cartilage cells but also regulated the expression of other growth factors in bone and cartilage tissue (28). PDGF and TGF- $\beta$ promoted the expression of vascular endothelial growth factor ${ }^{29)}$. In combination with other growth factors, IGF promoted differentiation and maturation of a variety of cells ${ }^{(30)}$. Okuda et al. ${ }^{(14)}$ reported that PRP stimulated osteoblast DNA synthesis and cell division. We contend that the combined effect of a variety of the high concentration of growth factors in PRP can stimulate osteogenesis and accelerate bone repair.

In the current study, we analyzed retrospectively the clinical efficacy of 16 recent cases (16 hips) of ONFH. According to the postoperative 3-month follow-up, none of the cases in either group showed disease progression, such as femoral head collapse, osteoarthritis requiring a total hip replacement, infections, or femoral neck fracture. In all patients, hip pain was significantly reduced or had disappeared.

There is no consensus in the literature on the potential role of PRP in promoting bone regeneration and repair. Whitman et al. (31) were the first to successfully use used PRP and bone transplantation to treat a bone defect in the field of maxillofacial surgery. Marx et al. (13) used PRP and a bone graft to treat a bone defect. They reported that the callus growth rate in the combined transplantation group was 1.62-2.16 times higher than that in a pure bone transplantation group. However, in a rabbit skull defect model Ahgaloo et al. ${ }^{(32)}$ found that PRP did not promote bone regeneration after an autologous bone graft. Choi et al. ${ }^{(33)}$ reported that PRP did not promote healing of bone defects 6 weeks later. Furthermore, in a study of PRP with an autologous bone composite, biological materials, and organic bone matrix, Kim et al. ${ }^{(34)}$ used and reported that PRP did not promote bone regeneration effectively.

The present study has several limitations. First, the follow-up time was relatively short. The study was performed only in the first 3 months after the surgery during the initial stage of rehabilitation. Second, the number of cases in the study was relatively small. Finally, the study was a retrospective case series.

\section{CONCLUSIONS}

In this comparison of a PRP group and controls in a clinical setting, the use of PRP was not associated with any obvious advantages. PRP eased hip pain and improved joint function. Long-term follow-up studies 
are needed to clarify the potential role of PRP in promoting repairing of $\mathrm{ONFH}$.

\section{ACKNOWLEDGEMENT}

This work was supported by the Science and Technology Development Planning of Shan Dong Province(2014GSF119022)

\section{CONFLICT OF INTEREST}

The authors declare no conflict of interests.

\section{REFERENCES}

1. Okazaki, S., Nagoya, S., Tateda, K., Katada, R., Mizuo, K., Watanabe, S., Yamashita, T., and Matsumoto, H. (2013) Experimental rat model for alcohol-induced osteonecrosis of the femoral head. International journal of experimental pathology 94, 312-319

2. Wu, Z., Ji, C., Li, H., Qiu, G., Gao, C., and Weng, X. (2013) Elevated level of membrane microparticles in the disease of steroid-induced vascular osteonecrosis. The Journal of craniofacial surgery 24, 1252-1256

3. Fukushima, W., Fujioka, M., Kubo, T., Tamakoshi, A., Nagai, M., and Hirota, Y. (2010) Nationwide epidemiologic survey of idiopathic osteonecrosis of the femoral head. Clinical orthopaedics and related research 468, 2715-2724

4. Chen, C. C., Lin, C. L., Chen, W. C., Shih, H. N., Ueng, S. W., and Lee, M. S. (2009) Vascularized iliac bonegrafting for osteonecrosis with segmental collapse of the femoral head. The Journal of bone and joint surgery. American volume 91, 2390-2394

5. Steinberg, M. E. (1988) Management of avascular necrosis of the femoral head--an overview. Instructional course lectures 37, 41-50

6. Garrigues, G. E., Aldridge, J. M., 3rd, Friend, J. K., and Urbaniak, J. R. (2009) Free Vascularized Fibular Grafting for treatment of osteonecrosis of the femoral head secondary to hip dislocation. Microsurgery 29, 342-345

7. Eppley, B. L., Woodell, J. E., and Higgins, J. (2004) Platelet quantification and growth factor analysis from platelet-rich plasma: implications for wound healing. Plastic and reconstructive surgery 114, 1502-1508

8. van den Dolder, J., Mooren, R., Vloon, A. P., Stoelinga, P. J., and Jansen, J. A. (2006) Platelet-rich plasma: quantification of growth factor levels and the effect on growth and differentiation of rat bone marrow cells. Tissue engineering 12, 3067-3073

9. Kon, E., Filardo, G., Delcogliano, M., Presti, M. L., Russo, A., Bondi, A., Di Martino, A., Cenacchi, A., Fornasari, P. M., and Marcacci, M. (2009) Plateletrich plasma: new clinical application: a pilot study for treatment of jumper's knee. Injury 40, 598-603

10. Wu, W., Chen, F., Liu, Y., Ma, Q., and Mao, T. (2007) Autologous injectable tissue-engineered cartilage by using platelet-rich plasma: experimental study in a rabbit model. Journal of oral and maxillofacial surgery : official journal of the American Association of Oral and Maxillofacial Surgeons 65, 1951-1957

11. Ahmad, M. A., Xypnitos, F. N., and Giannoudis, P. V. (2011) Measuring hip outcomes: common scales and checklists. Injury 42, 259-264

12. Feitosa, M. L., Fadel, L., Beltrao-Braga, P. C., Wenceslau, C. V., Kerkis, I., Kerkis, A., Birgel Junior, E. H., Martins, J. F., Martins Ddos, S., Miglino, M. A., and Ambrosio, C. E. (2010) Successful transplant of mesenchymal stem cells in induced osteonecrosis of the ovine femoral head: preliminary results. Acta cirurgica brasileira 25, 416-422

13. Marx, R. E., Carlson, E. R., Eichstaedt, R. M., Schimmele, S. R., Strauss, J. E., and Georgeff, K. R. (1998) Platelet-rich plasma: Growth factor enhancement for bone grafts. Oral surgery, oral medicine, oral pathology, oral radiology, and endodontics 85, 638-646

14. Okuda, K., Kawase, T., Momose, M., Murata, M., Saito, Y., Suzuki, H., Wolff, L. F., and Yoshie, H. (2003) Platelet-rich plasma contains high levels of platelet-derived growth factor and transforming growth factor-beta and modulates the proliferation of periodontally related cells in vitro. Journal of periodontology 74, 849-857

15. Landesberg, R., Roy, M., and Glickman, R. S. (2000) Quantification of growth factor levels using a simplified method of platelet-rich plasma gel preparation. Journal of oral and maxillofacial surgery : official journal of the American Association of Oral and Maxillofacial Surgeons 58, 297-300; discussion 300-291

16. Roldan, J. C., Jepsen, S., Miller, J., Freitag, S., Rueger, D. C., Acil, Y., and Terheyden, H. (2004) Bone formation in the presence of platelet-rich plasma vs. bone morphogenetic protein-7. Bone 34, 80-90

17. Weibrich, G., Hansen, T., Kleis, W., Buch, R., and Hitzler, W.E. (2004) Effect of platelet concentration in platelet-rich plasma on peri-implant bone regeneration. Bone 34, 665-671

18. Danesh-Meyer, M. J., Filstein, M. R., and Shanaman, R. (2001) Histological evaluation of sinus augmentation using platelet rich plasma (PRP): a 
case series. Journal of the International Academy of Periodontology 3, 48-56

19. Weibrich, G., Kleis, W. K., Hafner, G., and Hitzler, W. E. (2002) Growth factor levels in platelet-rich plasma and correlations with donor age, sex, and platelet count. Journal of cranio-maxillo-facial surgery : official publication of the European Association for Cranio-Maxillo-Facial Surgery 30, 97-102

20. Sanchez, A. R., Sheridan, P. J., and Kupp, L. I. (2003) Is platelet-rich plasma the perfect enhancement factor? A current review. The International journal of oral \& maxillofacial implants 18, 93-103

21. Bhanot, S., and Alex, J. C. (2002) Current applications of platelet gels in facial plastic surgery. Facial plastic surgery : FPS 18, 27-33

22. Yazawa, M., Ogata, H., Kimura, A., Nakajima, T., Mori, T., and Watanabe, N. (2004) Basic studies on the bone formation ability by platelet rich plasma in rabbits. The Journal of craniofacial surgery 15 , 439-446

23. Risau, W., Drexler, H., Mironov, V., Smits, A., Siegbahn, A., Funa, K., and Heldin, C. H. (1992) Platelet-derived growth factor is angiogenic in vivo. Growth factors (Chur, Switzerland) 7, 261266

24. Liu, Y., Zheng, W. K., Gao, W. S., Shen, Y., and Ding, W. Y. (2013) Function of TGF-beta and p38 MAKP signaling pathway in osteoblast differentiation from rat adipose-derived stem cells. European review for medical and pharmacological sciences 17, 1611-1619

25. Kitoh, H., Kitakoji, T., Tsuchiya, H., Mitsuyama, H., Nakamura, H., Katoh, M., and Ishiguro, N. (2004) Transplantation of marrow-derived mesenchymal stem cells and platelet-rich plasma during distraction osteogenesis--a preliminary result of three cases. Bone 35, 892-898

26. Lynch, S. E., Colvin, R. B., and Antoniades, H. N. (1989) Growth factors in wound healing. Single and synergistic effects on partial thickness porcine skin wounds. The Journal of clinical investigation 84, 640-646

27. Colciago, A., Celotti, F., Casati, L., Giancola, R., Castano, S. M., Antonini, G., Sacchi, M. C., and NegriCesi, P. (2009) In Vitro Effects of PDGF Isoforms (AA, BB, AB and CC) on Migration and Proliferation of SaOS-2 Osteoblasts and on Migration of Human Osteoblasts. International journal of biomedical science : IJBS 5, 380-389

28. Barrett, J. M., Rovedo, M. A., Tajuddin, A. M., Jilling, T., Macoska, J. A., MacDonald, J., Mangold, K. A., and Kaul, K. L. (2006) Prostate cancer cells regulate growth and differentiation of bone marrow endothelial cells through TGFbeta and its receptor, TGFbetaRII. The Prostate 66, 632-650

29. Petersen, W., Pufe, T., Zantop, T., Tillmann, B., and Mentlein, R. (2003) Hypoxia and PDGF have a synergistic effect that increases the expression of the angiogenetic peptide vascular endothelial growth factor in Achilles tendon fibroblasts. Archives of orthopaedic and trauma surgery 123, 485-488

30. Grohmann, M., Foulstone, E., Welsh, G., Holly, J., Shield, J., Crowne, E., and Stewart, C. (2005) Isolation and validation of human prepubertal skeletal muscle cells: maturation and metabolic effects of IGF-I, IGFBP-3 and TNFalpha. The Journal of physiology 568, 229-242

31. Whitman, D. H., Berry, R. L., and Green, D. M. (1997) Platelet gel: an autologous alternative to fibrin glue with applications in oral and maxillofacial surgery. Journal of oral and maxillofacial surgery : official journal of the American Association of Oral and Maxillofacial Surgeons 55, 1294-1299

32. Aghaloo, T. L., Moy, P. K., and Freymiller, E. G. (2002) Investigation of platelet-rich plasma in rabbit cranial defects: A pilot study. Journal of oral and maxillofacial surgery : official journal of the American Association of Oral and Maxillofacial Surgeons 60, 1176-1181

33. Choi, B. H., Im, C. J., Huh, J. Y., Suh, J. J., and Lee, S. H. (2004) Effect of platelet-rich plasma on bone regeneration in autogenous bone graft. International journal of oral and maxillofacial surgery 33, 56-59

34. Kim, S. G., Kim, W. K., Park, J. C., and Kim, H. J. (2002) A comparative study of osseointegration of Avana implants in a demineralized freeze-dried bone alone or with platelet-rich plasma. Journal of oral and maxillofacial surgery : official journal of the American Association of Oral and Maxillofacial Surgeons 60, 1018-1025 